\title{
Winning the DARPA Grand Challenge
}

\author{
Sebastian Thrun \\ Stanford University, Robotics Lab \\ thrun@stanford.edu
}

\begin{abstract}
The DARPA Grand Challenge has been the most significant challenge to the mobile robotics community in more than a decade. The challenge was to build an autonomous robot capable of traversing 132 miles of unrehearsed desert terrain in less than 10 hours. In 2004, the best robot only made 7.3 miles. In 2005, Stanford won the challenge and the $\$ 2 \mathrm{M}$ prize money by successfully traversing the course in less than 7 hours. This talk, delivered by the leader of the Stanford Racing Team, will provide insights in the software architecture of Stanford's winning robot. The robot massively relied on machine learning and probabilistic modeling for sensor interpretation, and robot motion planning algorithms for vehicle guidance and control. The speaker will explain some of the basic algorithms and share some of the excitement characterizing this historic event. He will also discuss the implications of this work for the future of the transportation.
\end{abstract}

